\title{
Excess protons in mesoscopic water-acetone nanoclusters
}

Rocío Semino, Jordi Martí, Elvira Guàrdia, and Daniel Laria

Citation: The Journal of Chemical Physics 137, 194301 (2012); doi: 10.1063/1.4766201

View online: https://doi.org/10.1063/1.4766201

View Table of Contents: http://aip.scitation.org/toc/jcp/137/19

Published by the American Institute of Physics

\section{Articles you may be interested in}

Excess protons in water-acetone mixtures

The Journal of Chemical Physics 136, 194503 (2012); 10.1063/1.4717712

Excess protons in water-acetone mixtures. II. A conductivity study

The Journal of Chemical Physics 139, 164510 (2013); 10.1063/1.4826464

The computer simulation of proton transport in water

The Journal of Chemical Physics 111, 9361 (1999); 10.1063/1.480032

Acetate ion and its interesting solvation shell structure and dynamics

The Journal of Chemical Physics 148, 094506 (2018); 10.1063/1.5019363

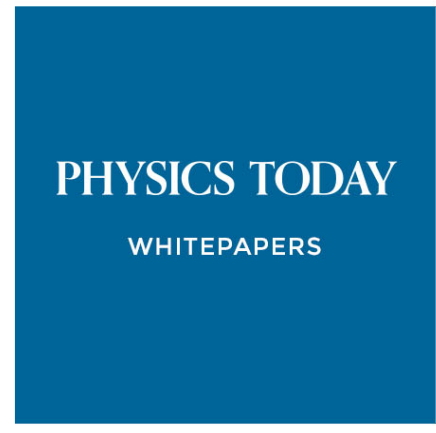




\title{
Excess protons in mesoscopic water-acetone nanoclusters
}

Rocío Semino, ${ }^{1}$ Jordi Martí, ${ }^{2}$ Elvira Guàrdia,${ }^{2}$ and Daniel Laria ${ }^{1,3, a)}$

${ }^{1}$ Departamento de Química Inorgánica Analítica y Química-Física e INQUIMAE,

Facultad de Ciencias Exactas y Naturales, Universidad de Buenos Aires, Ciudad Universitaria,

Pabellón II, 1428 Buenos Aires, Argentina

${ }^{2}$ Departament de Física i Enginyeria Nuclear, Universitat Politècnica de Catalunya-Barcelona Tech. B4-B5

Campus Nord, 08034 Barcelona, Spain

${ }^{3}$ Departamento de Física de la Materia Condensada, Comisión Nacional de Energía Atómica, Avenida Libertador 8250, 1429 Buenos Aires, Argentina

(Received 28 August 2012; accepted 21 October 2012; published online 19 November 2012)

\begin{abstract}
We carried out molecular dynamics simulation experiments to examine equilibrium and dynamical characteristics of the solvation of excess protons in mesoscopic, $[\mathrm{m}: n]$ binary polar clusters comprising $m=50$ water molecules and $n=6,25$, and 100 acetone molecules. Contrasting from what is found in conventional macroscopic phases, the characteristics of the proton solvation are dictated, to a large extent, by the nature of the concentration fluctuations prevailing within the clusters. At low acetone contents, the overall cluster morphology corresponds to a segregated aqueous nucleus coated by an external aprotic phase. Under these circumstances, the proton remains localized at the surface of the water core, in a region locally deprived from acetone molecules. At higher acetone concentrations, we found clear evidence of the onset of the mixing process. The cluster structures present aqueous domains with irregular shape, fully embedded within the acetone phase. Still, the proton remains coordinated to the aqueous phase, with its closest solvation shell composed exclusively by three water molecules. As the relative concentration of acetone increases, the time scales characterizing proton transfer events between neighboring water molecules show considerable retardations, stretching into the nanosecond time domain already for $n \sim 25$. In water-rich aggregates, and similarly to what is found in the bulk, proton transfers are controlled by acetone/water exchange processes taking place at the second solvation shell of the proton. As a distinctive feature of the transfer mechanism, translocation pathways also include diffusive motions of the proton from the surface down into inner regions of the underlying water domain. () 2012 American Institute of Physics. [http://dx.doi.org/10.1063/1.4766201]
\end{abstract}

\section{INTRODUCTION}

Protonated aqueous nanoclusters are common in nature. For example, they are ubiquitous in the upper atmosphere, where they host a rich variety of chemical processes with relevance in atmospheric chemistry. ${ }^{1,2}$ On the other hand, the analysis of these clusters provides information on a series of fundamental processes in solution chemistry, such as stepwise ionic solvation processes ${ }^{3,4}$ or size controlled acidbase equilibria ${ }^{5}$ and catalytic processes. ${ }^{6}$ Clusters of the type $\mathrm{H}^{+}\left(\mathrm{H}_{2} \mathrm{O}\right)_{n}$ with $n$, say, up to $\sim 30$ have been extensively analyzed from experimental ${ }^{7,8}$ and theoretical ${ }^{9-12}$ perspectives as well. Contrasting, the properties of mesoscopic aggregates, with linear dimensions intermediate between 10 and 100 Å remain somewhat less understood. ${ }^{13}$ The latter systems represent intermediate states with well differentiated structural and dynamical characteristics that cannot be straightforwardly interpolated from what is normally perceived in isolated-molecule states and in macroscopic phases. Still, the origin of many of these singularities can be normally traced back to the presence of a free interface in the close vicinity

\footnotetext{
a) Author to whom correspondence should be addressed. Electronic mail: dhlaria@cnea.gov.ar.
}

of a sizeable fraction of its components; however, in this size range, additional collective variables start to play key roles in determining their resulting structure and in controlling their chemical reactivity.

Binary, mixed polar protic/aprotic clusters combining water/acetonitrile or water/acetone provide clear examples: While, in the bulk, these solvents are miscible at all relative concentrations, there seems to be sufficient experimental evidence that would suggest that, in mesoscopic aggregates, they may phase separate. ${ }^{14,15}$ In a much broader context, demixing processes in nanoclusters ${ }^{16-18}$ would be just one example of a more general class of phenomena involving confinement in narrow slits ${ }^{19,20}$ or in the interior of nanotubes, ${ }^{21}$ to cite two relevant examples.

The analysis of protonated binary clusters gives rise to a series of new and interesting questions; most notably are those concerning the localization of the excess charge within the aggregate, which indirectly depends on the competitive solvation of the excess charge at different cluster sizes and compositions. In recent years, a large number of experimental and theoretical work has been undertaken to examine proton preferential solvation in mixed protic/aprotic clusters. The general picture that emerges from this body of research suggests that, beyond a relatively small number of water molecules, the 
proton exhibits propensity for aqueous solvation. This observation has been confirmed in mixed clusters combining water with acetonitrile, ${ }^{22}$ diethyl-ether, ${ }^{23}$ and acetone, ${ }^{24-27}$ and would clash with predictions based on the simple inspection of gas phase, proton affinities which for water turns out to be lower than the ones corresponding for the three aprotic species mentioned above. This feature underlines the importance of solvation as a key element determining the stable structures, even in aggregates comprising no more than, say, 10 molecules.

In the present paper we will present an analysis of proton solvation in mixed mesoscopic water/acetone clusters of different sizes based on molecular dynamics results. This study represents a natural extension of a previous molecular dynamics study, ${ }^{28}$ in which we addressed a similar analysis in bulk mixtures. As we will see, our simulations show that cluster results conjugate elements from our previous bulk analysis with those corresponding to the behavior of proton at macroscopic water/air, ${ }^{29-34}$ water/amphiphile solutions, ${ }^{35,36}$ and water/hydrophobic interfaces ${ }^{37}$ as well. In addition to this, proton states and the dynamical aspects connected to proton transfer events in clusters are also modulated by the characteristics of local concentration fluctuations which, in turn, derive from the overall boundary conditions that prevail in the systems under investigation.

The organization of this work is as follows: in Sec. II we will briefly describe the model system and the methodology that we implemented to perform the simulation experiments. Section III includes results for proton solvation structures within the different clusters, whereas dynamical aspects pertaining to proton transfer episodes in water-rich aggregates are presented in Sec. IV. The main conclusions of the study are summarized in Sec. V.

\section{MODEL}

We carried out molecular dynamics experiments on [m:n] binary nanoclusters, comprising $m$ molecules of water (W) and $n$ molecules of acetone (A) $\left[\left(\mathrm{CH}_{3}\right)_{2} \mathrm{CO}\right]$. Aqueous clusters with $m \sim 50$ represent perhaps the smallest aggregates in which one can unambiguously differentiate "surface" from "bulk" states. This will prove to be a key issue in the analysis of the proton solvation that will follow, so, in order be able to clearly identify such states, all simulation experiments were performed with $m$ fixed at 50 and $n=6,25$, and 100. A few test runs were also performed on [50:0] and [50:50] aggregates. When necessary, the component with the smaller (larger) mole fraction will be denoted as the solute (solvent).

Molecular dynamics trajectories for W-A systems were generated following a similar procedure to the one described in Ref. 28. Our approach is based on a large body of previous research performed by Voth and co-workers, so we will briefly describe its main features and refer the interested reader to Refs. 38 and 39 for additional details.

The dynamics of the classical nuclei with coordinates $\{\mathbf{R}\}$ and mass $M_{i}$ were derived from Newton's equation of motion applied on the potential energy surface
$\epsilon_{0}(\{\mathbf{R}(t)\}):$

$$
M_{i} \frac{\mathrm{d} \mathbf{R}_{i}(t)}{\mathrm{d} t}=-\nabla_{\mathbf{R}_{i}} \epsilon_{0}(\{\mathbf{R}(t)\}) .
$$

In the previous equation, $\epsilon_{0}(\{\mathbf{R}(t)\})=\left\langle\psi_{0}(t)\left|\hat{\mathrm{H}}_{\mathrm{EVB}}\right| \psi_{0}(t)\right\rangle$ represents the ground state potential energy surface of $\hat{\mathrm{H}}_{\mathrm{EVB}}$, a multi-state empirical valence bond (MS-EVB) Hamiltonian. ${ }^{38-42}$ Some basic ideas underlying the EVB formalism date back to works by Warshel and Weiss ${ }^{43,44}$ and rely on the assumption that the aqueous proton extends delocalized over different water molecules, giving rise to a base of diabatic states $\left\{\left|\phi_{i}\right\rangle\right\}$. As such, the different matrix elements of $\hat{\mathrm{H}}_{\mathrm{EVB}}$ expressed in this diabatic basis set, i.e., $h_{i j}(\{\mathbf{R}\})$ $=\left\langle\phi_{i}\left|\hat{\mathrm{H}}_{\mathrm{EVB}}\right| \phi_{j}\right\rangle$ depend exclusively on the instantaneous coordinates of the classical nuclei and are parametrized so as to bring EVB geometrical and energy results for a series of relevant $\mathrm{H}^{+}\left(\mathrm{H}_{2} \mathrm{O}\right)_{n}$ clusters, in reasonable agreement with highly refined quantum calculations.

At each step of the simulation procedure, the diabatic states were chosen according to a connectivity pattern of hydrogen bonds (HB) starting at the instantaneous pivot water molecule, denoted $\mathrm{H}_{3} \mathrm{O} *{ }^{39}$ After proper diagonalization, one obtains $\left|\psi_{0}\right\rangle$ and $\epsilon_{0}$, which can be expressed as

$$
\begin{gathered}
\left|\psi_{0}\right\rangle=\sum_{i=1}^{N_{\mathrm{EVB}}} c_{i}\left|\phi_{i}\right\rangle, \\
\epsilon_{0}(\{\mathbf{R}\})=\sum_{i, j} c_{i} c_{j} h_{i j}(\{\mathbf{R}\}) .
\end{gathered}
$$

The largest $c_{i}(t)$ determines the index of the instantaneous oxygen pivot which corresponds to the water molecule that exhibits stronger $\mathrm{H}_{3} \mathrm{O}^{+}$character. This index can be updated in the case of an eventual proton transfer event. Given the large acidity of the protonated-acetone cation in solution, one can safely disregard proton localization in A molecules. Additional details concerning intermolecular and intramolecular contributions of W-W, W-A, and A-A interactions incorporated in the parametrization of the matrix elements $h_{i j}$ are presented in Ref. 28 and references therein.

Clusters were generated from samples comprising appropriate amounts of $\mathrm{W}$ and $\mathrm{A}$, randomly distributed within spherical regions of radius $\sim 8-10 \AA$. The aggregates were equilibrated by multiple rescaling of the atomic velocities, following a thermal ramp with initial temperature $T_{i n}=100 \mathrm{~K}$ and final temperature $T_{f}=200 \mathrm{~K}$, along a $5 \mathrm{~ns}$ period. Atomic velocities were taken from Boltzmann distributions, after proper subtraction of the velocity of the center of mass and total angular momentum. At $T_{f}$, the clusters presented dynamical characteristics similar to those found in liquid phases and negligible evaporation. After the incorporation of the excess proton at central regions of the clusters, a second, $10 \mathrm{~ns}$ thermalization period at $T_{f}$ followed. Typically, the number of diabatic states considered in the diagonalization of $\hat{\mathrm{H}}_{\mathrm{EVB}}$ varied from $15-17$ at high $\mathrm{W}$ contents down to $\sim 4-5$ for [50:100] clusters. For each particular cluster size, statistics were harvested along three statistically independent microcanonical trajectories, each one lasting $\sim 30 \mathrm{~ns}$. Given the lengths of the simulation runs, and the inherent total energy jump episodes 
resulting from the restrictions in the number of diabatic states considered, special care was taken to maintain an adequate control of the energy conservation. To that end, we found it necessary to decrease the time steps down to $0.25 \mathrm{fs}$, to relax the hydrogen bond connectivity criterion when choosing diabatic states ${ }^{28}$ and expand the tree-like connectedness algorithm of hydrogen bonds so as to include patterns with bifurcated, double-donor and double-acceptor connectivity, as suggested in Ref. 45. With this procedure, we could maintain the energy conservation below, $3 \%-4 \%$ along the complete lengths of the simulation trajectories.

\section{CLUSTER EQUILIBRIUM SOLVATION STRUCTURES}

\section{A. Water as a solvent}

We will start our analysis by examining the characteristics of clusters at low-A contents. Perhaps the most direct route to gain a preliminary insight into the structural characteristics of these clusters, is the simple inspection of the snapshot shown in Fig. 1(a), which corresponds to a [50:25] cluster. At a first glance, it is clear that the gross structural characteristics of the aggregate can be cast in terms of a central water nucleus, coated with an A-external shell. Similar segregations involving aprotic solutes at cluster surfaces were

(a)

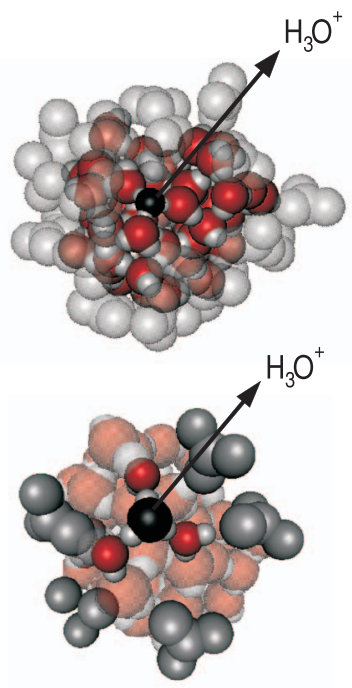

(c)

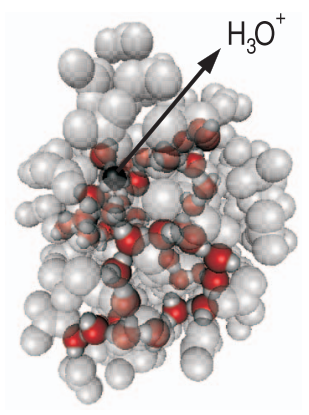

FIG. 1. Snapshots for selected [m:n] W-A nanoclusters. (a) [50:25]; (b) Top view of a [50:6] (see text); (c) [50:100]. For clarity purposes, A molecules are rendered in light gray (except in panel (b) that appear in dark gray). In the three panels, the pivot oxygen is rendered in black.

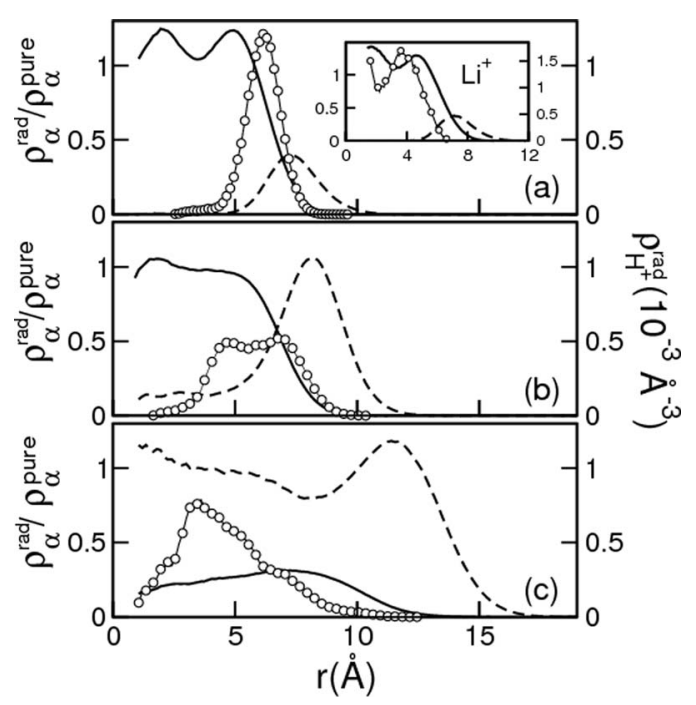

FIG. 2. Density fields for different species along the radial direction of the different [50:n] W-A clusters. Panels (a) $n=6$; (b) $n=25$; and (c) $n=100$. The solid lines correspond to oxygen sites in $\mathrm{W}$, the dashed lines correspond to oxygen sites in A whereas the white circles correspond to the oxygen pivot (see text). The $\mathrm{W}$ and A profiles appear normalized by the corresponding bulk densities (left $y$-axis); values for the pivot oxygen appear in the right $y$-axis.

reported in our previous study of mixed water/acetonitrile clusters. ${ }^{16}$

Spatial correlations involving different species along the cluster radial direction provide additional information based on more quantitative grounds. To facilitate our description, we found it convenient to define a local coordinate system centered at the centers of mass $(\mathrm{CM})$ of the different clusters. Panels (a) and (b) of Fig. 2 include results for density fields $\rho_{\alpha}^{\mathrm{rad}}(r)$, for [50:6] and [50:25] clusters, namely,

$$
\rho_{\alpha}^{\mathrm{rad}}(r)=\frac{1}{4 \pi r^{2}} \sum_{i=1}^{N_{\alpha}}\left\langle\delta\left(r_{i}^{\alpha}-r\right)\right\rangle
$$

in the previous equation, $\langle\cdots\rangle$ denotes a statistical average and $r_{i}^{\alpha}$ represents the distance between the position of the CM of $i$ th particle of species $\alpha\left(\alpha=\mathrm{W}, \mathrm{A}\right.$, and $\left.\mathrm{H}^{+}\right)$and the $\mathrm{CM}$ of the cluster; $N_{\alpha}=m(\alpha=\mathrm{W}), n(\alpha=\mathrm{A})$, and $1\left(\alpha=\mathrm{H}^{+}\right)$. The proton coordinate is defined by the expression, ${ }^{39}$

$$
\mathbf{r}^{\mathrm{H}^{+}}=\sum_{i}^{N_{\mathrm{EVB}}} c_{i}^{2} \mathbf{r}_{\mathrm{ds}}^{i}
$$

that takes into account the delocalization of the excess charge over the set of $N_{\mathrm{EVB}}$ different water molecules, with coordinates $\mathbf{r}_{\mathrm{ds}}^{i}$, associated to the $i$-diabatic state of $\hat{\mathrm{H}}_{\mathrm{EVB}}$. Note that the results for $\mathrm{W}$ and $\mathrm{A}$ (shown in the left $y$-axis) appear normalized by $\rho_{\alpha}^{\text {pure }}$, the corresponding bulk densities of the pure components, whereas the results for the excess proton are displayed in the right $y$-axis.

The $\mathrm{W}$ profile shown in panel (a) corresponds to a spherical-like, compact aggregate of radius $R \sim 6 \AA$, with density close to the standard bulk value of $\mathrm{W}$. Moreover, the plot reveals an internal shell structure in the nucleus, comprising a "bulk" shell at $r \sim 2 \AA$ and a "surface" shell $r \sim 5 \AA$. Beyond the latter distance, the profile falls smoothly down 
to zero, along a $\sim 2.5 \AA$ wide interface region, centered at $r \sim 6 \AA$. Contrasting, the curve describing spatial correlations of A molecules looks like a $3 \AA$ wide, Gaussian-like profile, centered at $r \sim 7 \AA$. Interestingly, the distribution corresponding to the excess charge also looks Gaussian, and its position practically coincides with that of the Gibbs-dividing surface of the underlying aqueous nucleus.

In principle, invoking elemental entropic arguments, the propensity of the excess charge to present surface solvation states could be somehow anticipated. However, this feature also highlights a "singular" characteristic of the proton in aqueous media. In this context the direct comparison with the solvation of a small size cation such as $\mathrm{Li}^{+}$is illustrative: In the inset of the top panel, we have included results for the density profiles corresponding to the solvation of $\mathrm{Li}^{+}$in a similar cluster. Note that, while the $\mathrm{W}$ and A curves show no meaningful modifications, the small cation does not lie at the water nucleus surface but mostly localizes in-between "bulk" and "surface" shells. The segregation of the proton at water/ air and water/hydrophobic interfaces is a well documented phenomenon and is the result of a complex interplay between, on the one hand, ion-water and water-water interaction and, on the other, geometrical aspects related to the charge distribution within the pivot. As such, there is a general consensus identifying the stabilization of the proton at the interface with the solvation structure which minimizes disruptions in the architecture of the water hydrogen bond network. ${ }^{29-34,37}$

Panel (b) of Fig. 2 includes plots of $\rho_{\alpha}^{\mathrm{rad}}(r)$ for [50:25] clusters. Although the gross structural features for these larger aggregates look similar to the ones shown in the top panel, a closer inspection reveals, at least, three relevant modifications: (i) a loss of structure in the internal water nucleus, (ii) a widening of its interface, and (iii) an incipient penetration of the external A phase deeper into the aqueous nucleus. Moreover, the increment of A molecules at the surface also promotes the penetration of the excess charge: Note that the corresponding plot now exhibits a double-peaked structure, suggesting a surface solvation state at $r_{\text {surf }}^{\mathrm{H}^{+}} \sim 7.5 \AA$ and an inner solvation state at $r_{\text {blk }}^{\mathrm{H}^{+}}$, located $3 \AA$ deeper into the water domain as well.

The presence of a compact, inner water nucleus and the localization of the proton mostly at its surface allows for assessing additional correlations involving orientational variables. Following previous analyses ${ }^{37}$ we will consider the following probability density:

$$
P_{\mu}(\cos \theta)=\left\langle\delta\left(\cos \theta_{\mu}-\cos \theta\right)\right\rangle
$$

with

$$
\cos \theta_{\mu}=\frac{\boldsymbol{\mu} \cdot \mathbf{r}^{\mathrm{O}^{*}}}{|\boldsymbol{\mu}| \mid \mathbf{r}^{\mathrm{O}^{*} \mid}} .
$$

In the previous equation, $\boldsymbol{\mu}$ corresponds to the dipolar moment of the charged $\left[\mathrm{H}_{3} \mathrm{O}\right]^{+}$moiety with respect to its $\mathrm{CM}$. Results for $P_{\mu}(\cos \theta)$ for the two clusters examined are displayed in the top panel of Fig. 3. For the [50:6] case, the distribution is sharply peaked at the vicinity of $\cos \theta=-1$, suggesting an antiparallel alignment of the hydronium dipolar moment with respect to the radial direction. Again, this observation is accordant with that reported in macroscopic
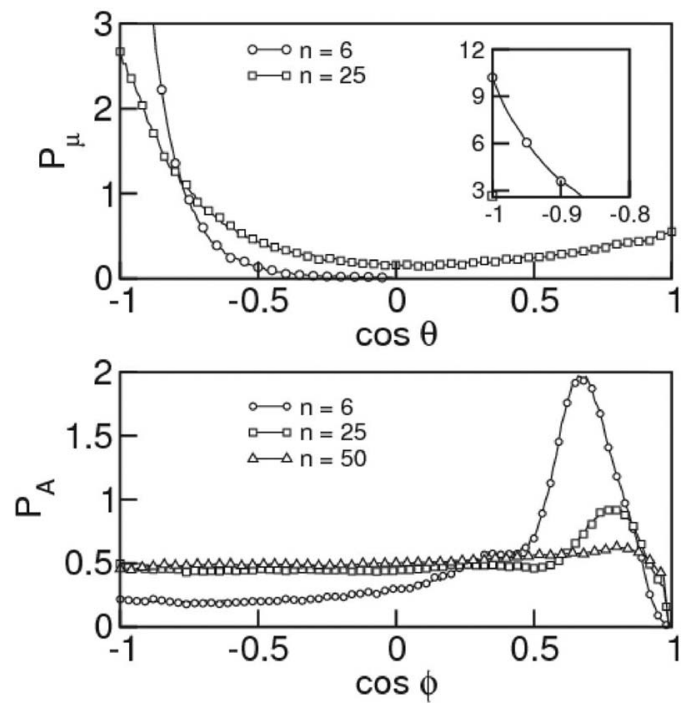

FIG. 3. Orientational correlations involving the solvation of excess proton in W-A clusters. Top panel: Angular distribution of the orientation of the dipole moment along radial directions (see text). Bottom panel: Angular distribution of the adsorbed A molecules at the underlying $\mathrm{W}$ nucleus with respect to the position of the excess charge.

water/air and water/hydrophobic interfaces. ${ }^{37}$ However, for [50:25] aggregates, the distribution widens significantly and a secondary, much milder maximum appears at $\cos \theta \sim 1$, indicating much rare episodes during which the previous alignment reverses.

The simultaneous analysis of the time evolutions of $r^{\mathrm{H}^{+}}(t)$ and $\cos \theta_{\mu}(t)$ along several nanoseconds (not shown) indicates that the latter inversion episodes coincide with time intervals during which $r^{\mathrm{H}^{+}}$remains close to $r_{\mathrm{blk}}^{\mathrm{H}^{+}}$. Moreover, the time evolution of $\cos \theta_{\mu}$ also shows that the pathways for the dipolar inversion involve gradual rotations of the $\left[\mathrm{H}_{3} \mathrm{O}\right]^{+}$ as it "plunges" into the water cluster and, in a few instances, sharp umbrella-inversions of the pyramidal structure of the hydronium as well. Similar inversion processes have been reported in a recent ab initio molecular dynamics of $\mathrm{H}_{3} \mathrm{O}^{+}\left(\mathrm{H}_{2}\right)_{n}$ clusters. ${ }^{46}$ We remark that we did not observe neither rotations nor inversion episodes for the case of [50:6] clusters, a fact that would suggest a higher degree of immobilization of the excess charge and its close environment at the cluster surface, compared to the situation in more internal regions of the water nucleus. ${ }^{37}$

The inspection of Fig. 1(b) will be helpful to introduce a second relevant orientational coordinate related with the distribution the A molecules at the $\mathrm{W}$ nucleus surface. The snapshot corresponds to a top view-with respect to the position of the proton- of a [50:6] cluster: One can clearly see a "partially capped" structure in which, at least, four out the six A molecules remain adsorbed in the close vicinity of the excess charge. Local surface density fluctuations of A molecules can be gauged quantitatively via the distribution $P_{\mathrm{A}}(\cos \phi)$ defined in terms of

$$
P_{\mathrm{A}}(\cos \phi)=\frac{1}{n} \sum_{i=1}^{n}\left\langle\delta\left(\cos \phi_{i}-\cos \phi\right)\right\rangle,
$$




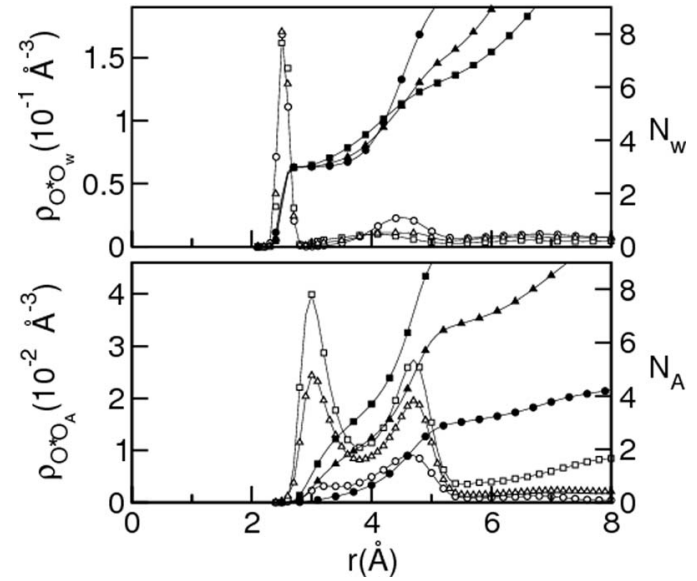

FIG. 4. Pair correlation functions (left $y$-axis) between the oxygen pivot and selected sites for mixed [50: $n$ ] clusters. Top panel: oxygen sites in W; bottom panel: oxygen sites in A. $n=6$ (circles); $n=25$ (triangles); and $n=100$ (squares). The corresponding cumulative integrals $N \alpha(r)$ are shown in black symbols (right $y$-axis).

where the angle $\phi_{i}$ is given by

$$
\cos \phi_{i}=\frac{\mathbf{r}^{\mathrm{H}^{+}} \cdot \mathbf{r}_{i}^{\mathrm{A}}}{\left|\mathbf{r}^{\mathrm{H}^{+}}\right|\left|\mathbf{r}_{i}^{\mathrm{A}}\right|} .
$$

Results for $P_{\mathrm{A}}(\cos \phi)$ are displayed in the bottom panel of Fig. 3 for three selected cluster sizes with $n=6,25$, and 50. At low surface coverages, the presence of a maximum for $\cos \phi \sim 0.67$ reveals a significant local enhancement of the A density next to the excess charge; meaningful surface density fluctuations still persist in [50:25] clusters, although the angular interval and their magnitudes are much more reduced. Finally, in clusters with $n=50$, one finds surface saturation and surface density fluctuations practically disappear at all relative orientations, except perhaps along the narrow $\cos \phi>0.9$ conical section, which remains deprived from A molecules.

The solvation structure in the close vicinity of the excess charge can be also analyzed via the usual pair correlation functions of the type:

$$
\rho_{\mathrm{O}^{*} \alpha}(r)=\frac{1}{4 \pi r^{2}} \sum_{i}\left\langle\delta\left(\left|\mathbf{r}_{i}^{\alpha}-\mathbf{r}^{\mathrm{O}^{*}}\right|-r\right)\right\rangle .
$$

Results for two relevant correlations involving oxygen sites in $\mathrm{W}\left(\alpha=\mathrm{O}_{\mathrm{W}}\right)$ and oxygen sites in $\mathrm{A}\left(\alpha=\mathrm{O}_{\mathrm{A}}\right)$ are shown in Fig. 4, where we have also included results for $N_{\alpha}(r)$, the corresponding cumulative integrals (black symbols, right $y$-axis). The constancy in the magnitudes and in the positions of the main peaks of $\rho_{\mathrm{O}^{*} \mathrm{O}_{\mathrm{W}}}(r)$, located at $r=2.55 \AA$, indicate that the two main features that characterize the short range coordination of excess protons in the bulk, persist in these aggregates. Here, we are referring to: (i) the tri-coordination via hydrogen bonds with water molecules in the first solvation shell and (ii) the absence of $\mathrm{H}-\mathrm{O}-\mathrm{H} \cdots\left[\mathrm{OH}_{3}\right]^{+}$-type acceptor bonding, due to the energetically unfavorable approach of external polar H-groups to the positively charged $\left[\mathrm{H}_{3} \mathrm{O}\right]^{+}$.

The coordination of the excess charge with the aprotic component also resembles the one found in the bulk and is characterized by a first peak at $r \sim 3 \AA$ and a secondary one located at $r \sim 4.8 \AA$. Contributions from the first peak in [50:6] clusters are clearly negligible, whereas in the [50:25] aggregates, correspond to molecules lying close to the "hydrophobic" region of the hydronium moiety, exposing their methyl groups. For both aggregates, the secondary peaks account for $\sim 3-4$ A molecules lying at the hydronium second solvation shell and acting as HB acceptors from the three water molecules located in the inner, first solvation shell. As we will see in the forthcoming section, these A molecules would play a key role controlling a possible mechanism for proton transfers between neighboring $\mathrm{W}$ molecules.

\section{B. Acetone as a solvent}

The structural analysis of A-rich clusters shows dramatic modifications which become self-evident by comparing snapshots (a) and (c) in Fig. 1: (i) first, there is a sensible loss of spherical symmetry in the overall shape of the clusters; (ii) more importantly, one can clearly observe incipient stages of the solubility of $\mathrm{W}$ in the aprotic phase. Concerning the last issue, the direct inspection of a set of trajectories reveals that local concentration fluctuations now involve irregular W domains, fully embedded within the A phase. The overall shapes of these irregular domains also present large fluctuations along a variety of lengthscales which eventually may become commensurate with the global cluster size, leading to frequent fragmentation and recombination episodes. The latter processes are the result of the alternance between compact and "thread-like" structures, during which a sensible reduction of the number of $\mathrm{W}-\mathrm{W}$ hydrogen bonding is observed.

Radial correlations involving the three relevant species are displayed in Fig. 2(c). Observe that the $\mathrm{W}$ profile has turned practically structureless and that the local density at the cluster center is now much more comparable to the one observed in bulk solutions with a similar overall molar fractions to that of the cluster, i.e., $\rho_{\mathrm{w}}^{\text {mix }}\left(x_{\mathrm{w}}=1 / 3\right) \sim 0.1 \rho_{\mathrm{w}}^{\text {pure }}$. Still, the $\mathrm{W}$ profile looks far from uniform. We can think of two reasons for such lack of uniformity: on the one hand, the already mentioned loss of sphericity in the overall cluster shape and, on the other, insufficient statistics to proper sampling slow modes associated with long-wavelength shape fluctuations of the domains. Concerning the A plot, it is clear that the aprotic component prevails at the outer region of the cluster, whereas in the central regions the curve levels off at a value also comparable to the one observed in the corresponding bulk mixture, i.e., $\rho_{\mathrm{A}}^{\text {mix }}\left(x_{\mathrm{w}}=1 / 3\right) \sim 0.9 \rho_{\mathrm{A}}^{\text {pure }}$. As per the proton is concerned, the analysis of its profile does not show any relevant new feature except, perhaps, the absence of surface solvation states, as a result of the break up of the original compact water nucleus. As will see in the next paragraph, the solvation structure of the excess charge will be very much controlled by its aqueous closest environment, so results for the proton density might still suffer from similar sampling deficiencies to those found for the $\mathrm{W}$ case.

The analysis of pair correlations functions (see top panel in Fig. 4) for these larger clusters shows that, even in those cases where $\mathrm{A}$ acts as the solvent, the Eigen cation ${ }^{47}$ $\left[\mathrm{H}_{3} \mathrm{O} \cdot\left(\mathrm{H}_{2} \mathrm{O}\right)_{3}\right]^{+}$remains the stable solvation structure of the 
excess charge at short distances. Note that, in the upper panel, the three plots are practically identical for $r<3.5 \AA$. Modifications in the local concentrations do appear at larger distances where $\mathrm{W}$ molecules get gradually replaced by A ones. In particular, in the lower panel, one observes that the integral under the secondary $\mathrm{O}^{*}-\mathrm{O}_{\mathrm{A}}$ peak accounts for $6 \mathrm{~A}$ molecules, saturating the second solvation shell coordinated with the first one via acceptor-donor HB partners. Moreover, we have verified that in the vast majority of the configurations, the Eigen cation represents the terminal link in one of the "string-like" aqueous domains (see Fig. 1(c)), and exhibits a high exposure to the aprotic phase.

Incidentally, the previous observations might lead us to compare the latter clusters with "spherical-like samples" of $x_{\mathrm{w}} \sim 0.3$, bulk solutions. However, we believe that such correspondence is likely to be misleading, due to the presence of non-negligible surface effects which promote, for example, the persistence of an external A-rich layer at the free interface. As such, a more realistic picture of these aggregates would be closer to a "sample of a liquid/air interface" of a protic/aprotic solution, in which similar external layer-like aprotic structures have been reported. ${ }^{48}$

\section{PROTON DYNAMICS IN CLUSTERS}

Our dynamical analysis will focus on proton transfer (PT) events in clusters. We start by considering population relaxations of the type,

$$
C(t)=\frac{\left\langle\delta h_{i}(t) \cdot \delta h_{i}(0)\right\rangle}{\left\langle\left(\delta h_{i}\right)^{2}\right\rangle},
$$

where $\delta h_{i}(t)=h_{i}(t)-\left\langle h_{i}\right\rangle$ represents the instantaneous deviation of the characteristic function $h_{i}$ at time $t$ from its average value. The dynamical variable $h_{i}(t)$ is equal to 1 if, at time $t$, the pivot oxygen corresponds to the $i$ th water molecule and 0 otherwise. According to Onsager's regression hypothesis, ${ }^{49}$ after an initial transient, $C(t)$ should exhibit an exponential decay, with characteristic time scale equal to the inverse of the proton rate, $k_{\mathrm{PT}}^{-1}=\tau_{\mathrm{PT}}$.

Results for $\ln C(t)$ for [50:6] and [50:25] clusters appear in Fig. 5 where, as a reference, we have included results for a [50:0] cluster as well. For [50:0] and [50:6], proton transfer characteristic time scales obtained from linear fits of $\ln C(t)$ for $t>50 \mathrm{ps}$ are in the order of $150-400 \mathrm{ps}$, whereas for [50:25] the predicted $\tau_{\mathrm{pt}}$ stretches into the nanosecond time scale (see Fig. 5 for the exact values). In passing, we remark that, for clusters with $n$ beyond $\sim 50$, we failed to detect PT events along the trajectories investigated. Leaving aside for the moment the obvious differences in temperature, these rates look considerably smaller, when compared to those obtained in bulk W-A solutions at ambient conditions. In the latter cases, the values of $k_{\mathrm{PT}}$ span from $\sim 0.1 \mathrm{ps}^{-1}$ for $x_{\mathrm{W}} \sim 0.8$ solutions, down to $\sim 0.01 \mathrm{ps}^{-1}$ for $x_{\mathrm{w}} \sim 0.2$ ones. Moreover, it is somehow unexpected that the incorporation of only $6 \mathrm{~A}$ molecules to a $50 \mathrm{~W}$ cluster may induce a reduction in the resulting rate by a factor of practically 3 .

To provide physically sound arguments to rationalize such modifications it will be instructive to pause for a moment and reconsider some conclusions about the controlling

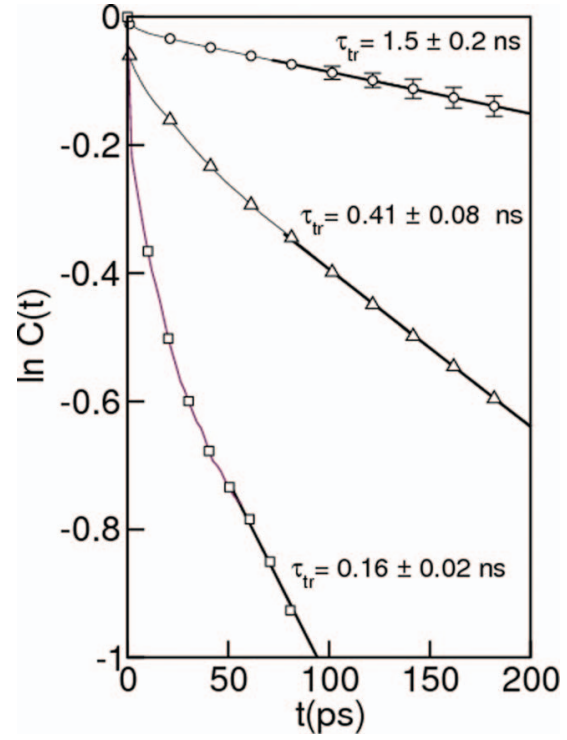

FIG. 5. Logarithm of the population relaxation of the pivot label for [50:n] W-A clusters. $n=0$ (squares); $n=6$ (triangles); and $n=25$ (circles). The dark solid lines correspond to linear fits for the $t>75$ ps decays.

mechanisms for proton transfers that we inferred from our previous analysis in W-A bulk solution. ${ }^{28}$ More specifically, we were able to detect that a key element that would make transfers possible would be an "appropriate" coordination of the eventual proton acceptor site. Such coordination gives rise to a symmetric solvation of a $\left[\mathrm{H}_{2} \mathrm{O} \cdot \mathrm{H} \cdot \mathrm{OH}_{2}\right]^{+}$Zundellike moiety, ${ }^{50}$ in which donor and acceptor partners are solvated on an equal foot, by two HB acceptor external water molecules. As such, PTs in W-A bulk solutions would be controlled by the exchange rates that prevail in the second solvation shell of the excess charge leading to such symmetric structures, which should become gradually more infrequent as the A concentration increases (see Fig. 6 in Ref. 28).

We now return to the [50:6] structural analysis: note that even though the global ratio $n / m \sim 0.1$ is a relatively small number, the local concentration of the aprotic solvent in the close vicinity of the excess charge was found to present a significant enhancement with respect to a uniform distribution of A molecules at the water nucleus surface (c.f. Fig. 3(b)). As a result, we verified that the cumulative integrals of $\rho_{\mathrm{O}^{*} \mathrm{O}_{\mathrm{A}}}$ for $n=6$ (see bottom panel of Fig. 4) already accounts for $\sim 3 \mathrm{~A}$ molecules lying the proton second solvation shell, and acting as $\mathrm{HB}$ acceptors from the Eigen moiety. Moreover, that number grows up to $\sim 4$ for [50:25] clusters and practically saturates at $\sim 6$ for $n>50$. This observation would explain the marked modifications in the proton rates operated by relatively moderate increments of $n$ and also, the absence of PT events in [50:50] and [50:100] clusters along temporal intervals of the order of $\sim 30-50 \mathrm{~ns}$.

Before closing this section we would like to also comment on a distinctive proton transfer mechanism that we were able to detect in [50:25] clusters. Figure 6 shows the time evolution of a few relevant physical observables during one illustrative example of such episodes. In the top panel, we display results for the pivot label, where we have arbitrarily set the 


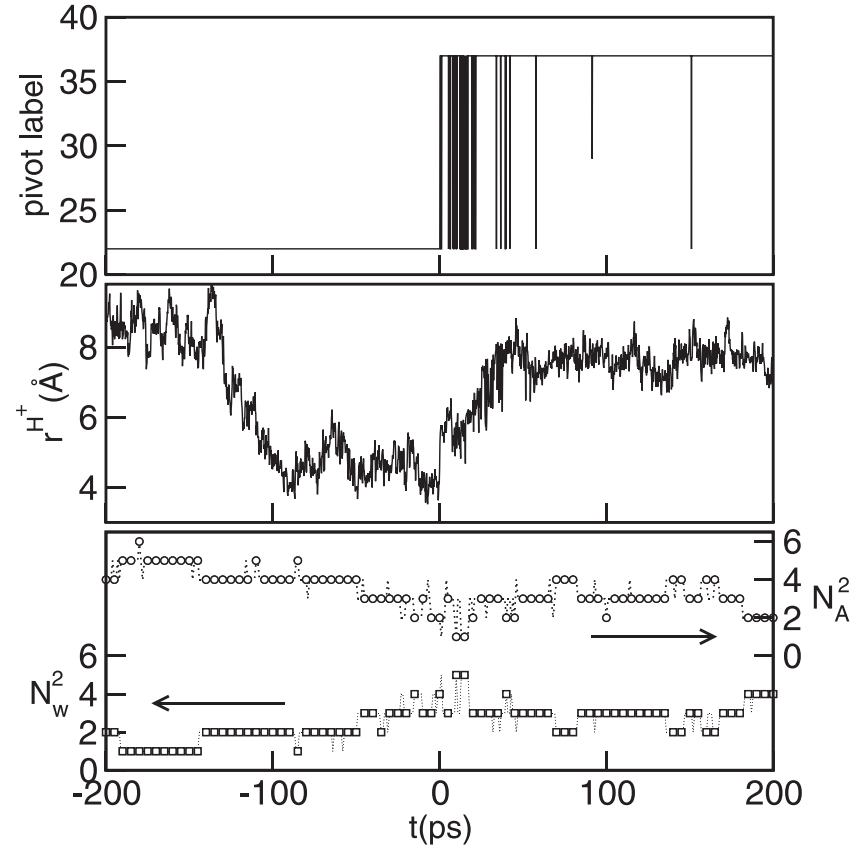

FIG. 6. Time evolution of a set of relevant observables along a proton transfer in [50:25] W-A nanoclusters. Top panel: pivot label; middle panel: distance of proton to the $\mathrm{CM}$ of the cluster; bottom panel: number of molecules of species $\alpha$ in the proton second solvation shell. W (squares, left $y$-axis); A (circles, right $y$-axis)

temporal origin at the beginning of a series of Zundel-like, high frequency resonances that anticipate the transfer from $\mathrm{W}$ molecule labeled 22 to the one labeled 37. Such resonances cease after a $\sim 20 \mathrm{ps}$ interval, beyond which the proton remains localized in the acceptor $\mathrm{W}$ site. In the middle panel, we present the time evolution of the distance between the CMs of the pivot and that of the cluster. Note that the PT event is preceded by an incursion of the pivot down into the water phase, as $r^{\mathrm{H}^{+}}$decreases from values close to $r_{\text {surf }}^{\mathrm{H}^{+}}$down to values $r_{\text {blk }}^{\mathrm{H}^{+}}$. A sharp, $\sim 2 \AA$ "jump" at $t=0$ can also be perceived, indicating that the initial transfer takes place outwards, along the radial direction. At $t \sim 50 \mathrm{ps,}$ and as the resonances die off, the new pivot "resurfaces," remaining localized at the cluster surface.

To complete this description, in the bottom panel, we present time evolutions of $N_{\alpha}^{2}(t)$, i.e., the number of molecules of species $\alpha(\alpha=\mathrm{W}, \mathrm{A})$ that lie in the pivot second solvation shell. Clearly, the proton transfer is made possible by the increment of $N_{\mathrm{W}}^{2}$ up to $\sim 6$ or, equivalently, by the concomitant reduction of $N_{\mathrm{A}}^{2}$ down to practically 0 . This modification is facilitated by the penetration of the pivot down into rich-W domains, a fact that would indicate a more complex pathway for PTs in clusters, in which the exchange at the second solvation shell would be preceded by diffusions of the excess charge into the water nucleus. In passing, we remark that the overall physical picture here would not differ substantially from what we have already reported in a previous analysis of proton transfer events in aqueous reverse micelles ${ }^{51}$ and would also agree with results from a recent analysis of proton diffusion at hydrophobic surfaces. ${ }^{52}$

\section{CONCLUDING REMARKS}

The analysis presented in this paper provides a new microscopic perspective on proton solvation states in mesoscopic clusters. Our molecular dynamics results confirm that, in mixed protic/aprotic binary aggregates, the solvation structure of the excess charge and its localization within the cluster are the result of a complex interplay between the prevailing concentration fluctuations and the competition for proton solvation between the two components.

The overall cluster structures present dramatic modifications which, to a large extent, are dictated by the relative concentrations of the components: At low A contentsin agreement with experimental evidence ${ }^{17,18}$ - the two fluids demix, giving rise to a central $\mathrm{W}$ nucleus, coated by an external A shell. Under these circumstances, the hydronium remains tightly bound to the surface of the water nucleus, with its rotational motions severely hindered, and exposing its "hydrophobic," lone-pair side to the bare water/air interface. As such, the resulting solvation structure resembles the one found in macroscopic water/air interfaces, ${ }^{29,31-34,37}$ and bulk methanol/water solutions. ${ }^{35,36}$

The gradual incorporation of aprotic molecules relaxes the structure of the interface of the aqueous nucleus and turns it wider. These effects allow for penetrations of the excess proton into the water domain and its marginal stabilization in inner aqueous shells. In the other limiting situation, i.e., at high A concentrations, the central W nucleus breaks up and the aqueous phase turns into a highly irregular, "thread"-like structure that spreads throughout most of the spatial extent of the cluster, exhibiting random fragmentation/recombination episodes.

From the dynamical side, we also found important changes in the temporal characteristics associated to proton transfer events between neighboring water molecules. For example, our results show that the incorporation of only a handful of aprotic molecules to a protonated aqueous cluster is sufficient to promote marked reductions in the characteristic rates describing translocations. To provide quantitative estimates, we found that the value of $\tau_{\text {tr }}$ for [50:25] aggregates differs by approximately one order from the corresponding value observed in pure water clusters and already falls in the nanosecond time domain. Moreover, for clusters with even larger A contents, the characteristic time scales seem to be exceedingly long to be detected in the course of simulation experiments lasting, say, a few tens of nanoseconds.

The analysis of local concentration fluctuations in the close vicinity of the proton provides clues to rationalize these dynamical modifications. As we mentioned in the previous paragraphs, we were unable to identify modifications in the first solvation shell of the proton. Yet, we did detect changes in the second one and beyond. For example, our results show that, in [50:6] clusters, already one half of the total number of acceptor sites in this shell, is occupied by A molecules, a result comparable to the one found in $x_{\mathrm{w}} \sim 0.5$ bulk solutions. This large enhancement in the A population represents the response of the aprotic molecules to the Coulomb coupling with the excess charge. Note that these molecules, in turn, act as spacers, inhibiting a sizeable fraction of the reactive 
pathways available for proton translocations. For the largest cluster investigated, $N_{\mathrm{A}}^{2} \sim 5-6$, and proton transfer events become much rare events. Under these circumstances, one could speculate that the eventual increments in $N_{\mathrm{w}}^{2}$ that would make transfers possible, could be controlled by longwavelength, slow dynamical modes, involving global shape reorganizations of the aqueous domains, that we failed to detect.

Finally, we would like to comment that the consideration of a series of reactive proton transfer events in clusters with intermediate acetone concentrations showed a correlation between these events and diffusive motions of the excess charge, into the underlying water nucleus. Such displacements would reveal that, in addition to the exchange between aprotic and protic components in the second solvation shell of the hydronium, the mechanisms controlling proton transfer pathways in these clusters should include additional elements reflecting couplings with dynamical modes of the free interface.

Still, the description presented here is far from being complete. For example, at temperatures of the order of $\sim 200 \mathrm{~K}$ quantum effects on the proton structural and dynamical descriptions are likely to be non-negligible. From previous analysis, ${ }^{39}$ we can anticipate that our predictions for the fluctuations in spatial correlations and for $\tau_{\text {tr }}$ are probably overestimated. However, we remain confident that the present analysis provides new and physically sound insights that will be useful to predict and to rationalize relevant experimental information about chemical reactivity involving protonated species in mixed mesoscopic phases.

\section{ACKNOWLEDGMENTS}

Financial support from the Sabbatical Program for Foreign Scientists sponsored by the Ministerio de Educación, Cultura y Deporte de España is very much appreciated. E.G. and J.M. gratefully acknowledge financial support from the Direcció General de Recerca de la Generalitat de Catalunya (Grant No. 2009-SGR-1003) and the Spanish MICINN (Grant No. FIS2009-13641-C02-01). D.L. is a staff member of CONICET-Argentina.

${ }^{1}$ R. P. Wayne, Chemistry of Atmospheres (Oxford University Press, Cambridge/New York, 2000).

${ }^{2}$ R. G. Keese and A. W. Castleman, Jr., J. Geophys. Res. 90, 5885, doi:10.1029/JD090iD04p05885 (1985).

${ }^{3}$ N. F. Dalleska, K. Honma, and P. B. Armentrout, J. Am. Chem. Soc. 115, 12125 (1993).

${ }^{4}$ M. D. Tissandier, K. A. Cowen, W. Y. Feng, E. Cundlach, M. H. Cohen, A. D. Eachart, J. V. Coes, and T. R. Tuttle, J. Phys. Chem. A 102, 7787 (1998).

${ }^{5}$ V.-A. Glezakou, M. Dupuis, and C. J. Mundy, Phys. Chem. Chem. Phys. 9, $5752(2007)$.

${ }^{6}$ V. Vaida, J. Chem. Phys. 135, 020901 (2011).

${ }^{7}$ G. Niedner-Schatteburg and V. E. Bondybey, Chem. Rev. 100, 4059 (2000).

${ }^{8}$ E. Dermota, Q. Zhong, and A. W. Castleman, Jr., Chem. Rev. 104, 1861 (2004).

${ }^{9}$ E. Kochanski, R. Kelterbaum, S. Klein, M. M. Rohmer, and A. Rahmouni, Adv. Quantum Chem. 28, 273 (1997).

${ }^{10}$ X. C. Huang, H. M. Cho, S. Carter, L. Ojamae, J. M. Boaman, and S. J. Singer, J. Phys. Chem. A 107, 7142 (2003).

${ }^{11}$ C. J. Burnham, M. K. Petersen, T. J. F. Day, S. S. Iyengar, and G. A. Voth, J. Chem. Phys. 124, 02437 (2006)

${ }^{12}$ S. S. Iyengar, M. K. Petersen, T. J. Day, C. J. Burnham, V. E. Teige, and G. A. Voth, J. Chem. Phys. 123, 084309 (2005).
${ }^{13}$ H.-C. Chang, C.-C. Wu, and J.-L. Kuo, Int. Rev. Phys. Chem. 24, 553 (2005).

${ }^{14}$ M. Ahmed, C. J. Apps, C. Hughes, N. E. Wat, and J. C. Whitehead, J. Phys. Chem. A 101, 1250 (1997).

${ }^{15}$ A. Wakisaka, H. Adboul-Carime, Y. Yamamoto, and Y. Kiyozumi, J. Chem. Soc., Faraday Trans. 94, 369 (1998).

${ }^{16}$ V. Molinero, D. Laria, and R. Kapral, J. Chem. Phys. 109, 6844 (1998).

${ }^{17}$ T. Schindler, C. Berg, G. Niedner-Schatteburg, and V. E. Bondybey, Chem. Phys. 201, 491 (1995).

${ }^{18}$ M. M. Y. Lyktey, R. L. DeLeon, K. S. Shores, T. Furlani, and J. F. Garvey, J. Phys. Chem. A 104, 5197 (2000).

${ }^{19}$ S. Kittaka, M. Kuranishi, S. Ishimaru, and O. Umahara, J. Chem. Phys. 126, 091103 (2007).

${ }^{20}$ J. Rodriguez, M. D. Elola, and D. Laria, J. Phys. Chem. B 113, 12744 (2009).

${ }^{21}$ J. Rodriguez, M. D. Elola, and D. Laria, J. Phys. Chem. B 114, 7900 (2010).

${ }^{22}$ C. A. Deakyne, M. Meot-Ner (Mautner), C. L. Campbell, M. G. Hughes, and S. P. Murphy, J. Chem. Phys. 84, 4958 (1986).

${ }^{23}$ H.-C. Chang, H.-C. Jiang, I. Hahndorf, S. H. Lin, Y. T. Lee, and H.-C. Chang, J. Am. Chem. Soc. 121, 4443 (1999).

${ }^{24}$ W. Achats, S. Joos, C. Berg, T. Schindler, M. Beyer, G. Albert, G. NiednerSchatteburg, and V. W. Bodybey, J. Am. Chem. Soc. 120, 1876 (1998).

${ }^{25}$ Y. Kawai, S. Yamaguchi, Y. Okada, and K. Takeuchi, Int. J. Mass. Spectrom. 220, 375 (2002).

${ }^{26}$ M. Meot-Ner (Mautner), S. Scheiner, and W. O. Yu, J. Am. Chem. Soc. 120, 6980 (1998).

${ }^{27}$ S. Wei, W. B. Tzeng, R. G. Keese, and A. W. Castleman, Jr., J. Am. Chem. Soc. 113, 1960 (1991).

${ }^{28}$ R. Semino and D. Laria, J. Chem. Phys. 136, 194503 (2012).

${ }^{29}$ M. K. Petersen, S. S. Iyengar, T. J. F. Day, and G. A. Voth, J. Phys. Chem. B 108, 14804 (2004).

${ }^{30}$ S. S. Iyengar, T. J. F. Day, and G. A. Voth, Int. J. Mass Spectrom. 241, 197 (2005).

${ }^{31}$ M. Mucha, T. Frigato, L. M. Levering, H. C. Allen, D. J. Tobias, L. X. Dang, and P. Jungwirth, J. Phys. Chem. B 109, 7617 (2005).

${ }^{32}$ R. Vácha, V. Buch, A. Milet, J. P. Devlin, and P. Jungwirth, Phys. Chem. Chem. Phys. 9, 4736 (2007).

${ }^{33}$ B. Jagoda-Cwiklik, L. Cwiklik, and P. Jungwirth, J. Phys. Chem. A 115, 5881 (2011).

${ }^{34}$ H. Takahasi, K. Maruyama, Y. Karino, A. Morita, M. Nakano, P. Jungwirth, and N. Matubayasi, J. Phys. Chem. B 115, 4745 (2011).

${ }^{35}$ M. K. Petersen and G. A. Voth, J. Phys. Chem. B 110, 7085 (2006).

${ }^{36}$ J. A. Morrone, K. E. Haslinger, and M. E. Tuckerman, J. Phys. Chem. B 110, 3712 (2006).

${ }^{37}$ S. Iuchi, H. Chen, F. Paesani, and G. A. Voth, J. Phys. Chem. B 113, 4017 (2009)

${ }^{38}$ U. W. Schmitt and G. A. Voth, J. Phys. Chem. B 102, 5547 (1998).

${ }^{39}$ U. W. Schmitt and G. A. Voth, J. Chem. Phys. 111, 9361 (1999).

${ }^{40} \mathrm{R}$. Vuilleumier and D. Borgis, in Classical and Quantum Dynamics in Condensed Phase Simulations, edited by B. J. Berne, G. Ciccotti, and D. F. Coker (World Scientific, Singapore, 1998), Chap. 30.

${ }^{41}$ G. A. Voth, Acc. Chem. Res. 39, 143 (2006).

${ }^{42}$ C. Knight and G. A. Voth, Acc. Chem. Res. 45, 101 (2012).

${ }^{43}$ A. Warshel and R. M. Weiss, J. Am. Chem. Soc. 102, 6218 (1980).

${ }^{44} \mathrm{~A}$. Warshel, Computer Modelling of Chemical Reactions in Enzymes and Solutions (Wiley, New York, 1991).

${ }^{45}$ Y. Wu, H. Chen, F. Wang, F. Paesani, and G. A. Voth, J. Phys. Chem. B 112, 467 (2008).

${ }^{46}$ M. Baer, D. Marx, and G. Mathias, ChemPhysChem 12, 1906 (2011).

${ }^{47}$ M. Eigen and L. De Maeyer, Proc. R. Soc. London, Ser. A 247, 505 (1958).

${ }^{48}$ Similar concentration enhancements have been reported at the bare interface of water/acetonitrile bulk solutions. See, for example, D. Zhang, J. H. Gutow, K. B. Eisenthal, and T. F. Henz, J. Chem. Phys. 98, 5099 (1993); Y. Rao, N. J. Turro, and K. B. Eisenthal, J. Phys. Chem. C 113, 14384 (2009); L. B. Pártay, P. Jedlovszky, and G. Horvai, ibid. 113, 18173 (2009).

${ }^{49} \mathrm{See}$, for example, D. Chandler, in Introduction to Modern Statistical Mechanics (Oxford University Press, New York, 1987), Chap. 8.

${ }^{50}$ G. Zundel and H. Metzger, Z. Phys. Chem. 58, 225 (1968).

${ }^{51}$ J. Rodriguez, J. Martí, E. Guàrdia, and D. Laria, J. Phys. Chem. B 111, 4432 (2007).

${ }^{52}$ C. Zhang, D. G. Knyazev, Y. A. Vereshaga, E. Ippoliti, T. H. Nguyen, P. Carloni, and P. Pohl, Proc. Natl. Acad. Sci. U.S.A. 109, 9744 (2012). 